\title{
PROCEEDINGS
}

\section{A generalized Coleman-Noll procedure for the exploitation of the entropy principle}

\author{
By V. A. Cimmelli*, A. Sellitto and V. Triani \\ Department of Mathematics and Computer Science, University of Basilicata, \\ Campus Macchia Romana, Viale dell'Ateneo Lucano, 10, Potenza 85100, Italy
}

A generalization of the classical Coleman-Noll procedure for the exploitation of second law of thermodynamics in the presence of first-order non-local constitutive functions is proposed. The local balance of entropy is regarded as a differential inequality constrained by the governing equations for the set of the unknown fields as well as by their gradient extensions. The thermodynamic compatibility of such a class of materials is achieved without any modification of the basic thermodynamic laws. The results so obtained are applied to model nonlinear heat conduction in solids, in the presence of a dynamical semi-empirical temperature scale.

Keywords: non-equilibrium continuum thermodynamics; exploitation of second law; extended Coleman-Noll procedure; weakly non-local heat conduction in rigid solids

\section{Introduction}

In continuum thermodynamics, the entropy principle constitutes a basic tool in order to derive thermodynamic restrictions on the constitutive functions (Truesdell 1984). In literature, one can find two rigorous mathematical procedures, the Coleman-Noll (Coleman \& Noll 1963) and the Liu one (Liu 1972), which are faced with the problem of finding necessary and sufficient conditions ensuring that the dissipation inequality is satisfied in correspondence of arbitrary solutions of the governing equations for the wanted fields. The ColemanNoll procedure was developed in the framework of rational thermodynamics (Truesdell 1984). In such an approach, for rigid heat conductors, the second law of thermodynamics is expressed locally by the Clausius-Duhem inequality

$$
-(\dot{\psi}+s \dot{\theta})-\frac{1}{\theta} q_{i} \theta_{, i} \geq 0,
$$

where $\theta$ is the absolute temperature, $\psi=(e-\theta s)$ is the Helmholtz free energy per unit of volume, $e$ being the internal energy density, $s$ is the entropy density and $q_{i}$, $i=1,2,3$ are the components of the heat flux q. In equation (1.1), a superposed dot denotes the derivative with respect to time, the symbol $\left(,{ }_{i}\right)$ denotes partial differentiation with respect to the current cartesian coordinates $x_{i}$, the repeated

*Author for correspondence (vito.cimmelli@unibas.it).

In memory of Antonio Mario Tamburro, Rector Magnificus of the University of Basilicata. 
indices implying summation. In the absence of heat sources, the inequality (1.1) is obtained by substituting the local balance of energy

$$
\dot{e}+q_{i, i}=0,
$$

into the entropy inequality

$$
\sigma_{s}=\dot{s}+\left(\frac{q_{i}}{\theta}\right)_{, i} \geq 0,
$$

where $\sigma_{s}$ is the local entropy production.

It is worth observing that, in such a way, the differential constraint represented by the balance of energy is taken into account only quantitatively but not qualitatively, because its role is the same whatever the class of materials is. This is a consequence of the fact that the space and time derivatives appearing therein are not developed and hence the constitutive equations cannot be taken into account.

Rational thermodynamics introduces the gradients of the unknown fields as arguments of the constitutive functions (weak non-locality). This represents a useful mean in modelling phenomena in which the behaviour of the material in a point depends on its interactions with the closest particles too. Weakly nonlocal constitutive equations are mostly investigated in the study of the material microstructure (Capriz 1989), or in the gradient extension of classical theories (Cimmelli \& Frischmuth 2007).

If the gradients enter the constitutive equations of deformable continua, and the balance of energy is accepted in its classical form (Coleman \& Noll 1963), the Coleman-Noll procedure leads to the consequence that the entropy, the free energy and the other thermodynamic potentials cannot depend on the gradients, whatever is the order of non-locality of the constitutive equations. Such a conclusion may generate serious discrepancies, because it renders some important classes of non-local materials, such as the Korteweg fluids (Korteweg 1901), incompatible with the second law (Dunn \& Serrin 1985; Cimmelli et al. $2009 b$ ). One can mention several other situations in which the entropy is required to depend on the gradients of the unknown variables as, for instance, damage theory (Bazant \& Pijauder-Cabot 1987; Ván \& Vásárhelyi 2001), liquid crystals (Papenfuss \& Forest 2006) and critical phenomena at low temperature (Lebon et al. 1998).

In order to circumvent these problems, and still remain in the framework of rational thermodynamics, two different approaches can be found in literature.

The first one modifies the entropy inequality (Müller 1967), by postulating the existence of an entropy extra-flux $\mathbf{k}$ such that the total entropy flux becomes $\mathbf{J}=(\mathbf{q} / \theta)+\mathbf{k}$. The second one modifies the local balance of energy, by supposing the existence of an energy extra-flux $\mathbf{u}$, owing to the matter diffusion (Gurtin \& Vargas 1971) or to the interstitial working of long-range interactions (Dunn \& Serrin 1985). However, even if we assume one of the two points of view above, problems arise in dealing with the first-order non-local constitutive equations. This is due to the fact that the Coleman-Noll procedure allows the entropy to depend on the gradients of order $n$ of the unknown fields if, and only if, the constitutive functions depend on the gradients of order $n+1$. In the case of first-order non-locality, it is clear that this leads again to a local entropy (Müller 1967; Gurtin \& Vargas 1971; Dunn \& Serrin 1985). 
Recently, the above problem has been afforded by Cimmelli (2007) from a different point of view. His proposal was that one should consider as constraints for the entropy inequality both the governing equations of the wanted fields and their gradient extensions up to the order which appears in the state space. Starting from this idea, an extension of the classical Liu procedure (Liu 1972) was developed. The new methodology has been applied in modelling second-grade Korteweg-type fluids (Cimmelli et al. 2009b) and non-local effects and second sound in solids (Cimmelli et al. 2009a). In both cases, the entropy is non-local, without the needing of any extra-flux.

In the present paper, we propose a generalization of the classical Coleman-Noll procedure which is in accordance with the above point of view.

Our proposal is that one should substitute into the inequality (1.1) the governing equations of the wanted fields, and their gradient extensions up to the order which appears in the state space, so that the number of the constraints considered is every equal to the number of independent thermodynamic variables. Moreover, these equations should be substituted in such a way that the time derivatives of the thermodynamic potentials and the divergences of the fluxes should be explicitly calculated, so that the influence of the constitutive equations on the form of the dissipation inequality should emerge. Indeed, a serious problem arising in applying the generalized procedure illustrated above is that the substitution of the differential constraints into the entropy inequality can be realized in several ways, giving rise to different expressions for the dissipation inequality. Our proposal above provides a criterion which should be taken into account in order to obtain the most appropriate substitution.

In $\S 2$, we consider a general scheme in which the constitutive functions are admitted to depend on the absolute temperature $\theta$, on a set of intensive variables $\mathbf{z}$ ruled by a governing equation which is not in the balance form, on a set of extensive variables $\mathbf{w}$, for which a balance equation is assigned, and on the first-order gradients of all the aforementioned variables. Then, by substituting in equation (1.1) the governing equations of the wanted fields and their gradient extensions, the thermodynamic restrictions on the constitutive functions are obtained starting by the observation that such an extended inequality is linear in a suitable set of space and time derivatives which are considered to be completely arbitrary. The main consequence of the new extended approach is that a non-local entropy is compatible with the restrictions placed by the second law, against the commonly accepted statement that first-order spatial non-localities do not influence the thermodynamic potentials but contribute only to the fluxes.

In $\S 3$, we apply the general scheme of $\S 2$ in modelling weakly non-local and nonlinear heat transport in solids. We consider a rigid heat conductor whose state space is spanned by the absolute temperature $\theta$, a dynamical semi-empirical temperature $\beta$ (Cimmelli \& Kosiński 1991) and their first-order gradients. A governing equation for $\beta$ is assigned. It is shown that the non-local terms entering the thermodynamic potentials and the evolution equation of $\beta$ lead to a nonlinear extension of the celebrated Guyer-Krumhansl heat transport equation (Guyer \& Krumhansl 1966).

Finally, in $\S 4$, concluding remarks together with the possible further developments of the theory are presented. 


\section{Extended Coleman-Noll inequality}

Let us consider heat conducting rigid bodies whose constitutive functions are defined on the set

$$
Z=\left\{\theta, \theta_{, i}, z_{\alpha}, z_{\alpha, i}, w_{A}, w_{A, i}\right\}
$$

with $\alpha=1, \ldots, \bar{N}$, and $A=1, \ldots, \bar{M}$. Let us notice that in equation (2.1), two additional types of indices have been used: the Greek index and the capital Latin one. The former means the generic component of $\mathbf{z}$ and runs from 1 to $\bar{N}$. The latter, instead, means the generic component of $\mathbf{w}$ and runs from 1 to $\bar{M}$.

Let us suppose that the components $z_{\alpha}$ of the intensive state variable $\mathbf{z}$ are ruled by an evolution equation of the type

$$
\dot{z}_{\alpha}=f_{\alpha}^{0}(Z) \dot{\theta}+f_{\alpha \beta i j}^{1}(Z) z_{\beta, i j}+f_{\alpha B i j}^{2}(Z) w_{B, i j},
$$

wherein $f^{0}, f^{1}$ and $f^{2}$ are suitable tensorial functions.

The components $w_{A}$ of the extensive state variable $\mathbf{w}$ are instead governed by a balance law

$$
\dot{w}_{A}+\Phi_{A j, j}=0,
$$

where $\Phi_{A j}$ is the $j$-th component of the flux term and, for the sake of simplicity, the production of $w_{A}$ has been supposed to be zero.

It is worth observing that an evolution equation for the temperature $\theta$ can be obtained by taking into account equations (1.1) and (2.1). In the absence of heat sources, it reads

$$
\begin{aligned}
c \dot{\theta} & +\frac{\partial e}{\partial \theta_{, j}} \dot{\theta}_{, j}+\frac{\partial e}{\partial z_{\beta}} \dot{z}_{\beta}+\frac{\partial e}{\partial z_{\beta, k}} \dot{z}_{\beta, k}+\frac{\partial e}{\partial w_{A}} \dot{w}_{A}+\frac{\partial e}{\partial w_{A, k}} \dot{w}_{A, k} \\
& +\frac{\partial q_{i}}{\partial \theta} \theta_{, i}+\frac{\partial q_{i}}{\partial \theta_{, j}} \theta_{, j i}+\frac{\partial q_{i}}{\partial z_{\beta}} z_{\beta, i}+\frac{\partial q_{i}}{\partial z_{\beta, k}} z_{\beta, k i}+\frac{\partial q_{i}}{\partial w_{A}} w_{A, i}+\frac{\partial q_{i}}{\partial w_{A, k}} w_{A, k i}=0
\end{aligned}
$$

with $c=\partial e / \partial \theta$ as the specific heat. The above equation constitutes the constraint on the state variable $\theta$, to be taken into account in deriving the consequences of the entropy principle.

Owing to equation (2.1), the inequality (1.1) reads

$$
\left(\frac{\partial \psi}{\partial \theta}+s\right) \dot{\theta}+\frac{\partial \psi}{\partial \theta_{, j}} \dot{\theta}_{, j}+\frac{\partial \psi}{\partial z_{\beta}} \dot{z}_{\beta}+\frac{\partial \psi}{\partial z_{\beta, k}} \dot{z}_{\beta, k}+\frac{\partial \psi}{\partial w_{A}} \dot{w}_{A}+\frac{\partial \psi}{\partial w_{A, k}} \dot{w}_{A, k}+\frac{1}{\theta} q_{i} \theta_{, i} \leq 0 .
$$

Our next step consists in obtaining a very general entropy inequality, the extended Coleman-Noll inequality, by substituting into equation (2.5) the differential constraints (2.2)-(2.4), together with their spatial gradients. After some lengthy calculations, we get

$$
\begin{aligned}
& {\left[\frac{\partial \psi}{\partial z_{\alpha}} f_{\alpha}^{0}+\frac{\partial \psi}{\partial z_{\alpha, i}}\left(f_{\alpha}^{0}\right)_{, i}-\frac{1}{c} \frac{\partial \psi}{\partial \theta_{, i}}\left(\frac{\partial e}{\partial \theta}\right)_{, i}\right]_{\dot{\theta}}} \\
& \quad-\left[\frac{1}{c}\left(\frac{\partial \psi}{\partial \theta}+s\right) \frac{\partial e}{\partial \theta_{, j}}+\frac{1}{c} \frac{\partial \psi}{\partial \theta_{, i}}\left(\frac{\partial e}{\partial \theta_{, j}}\right)_{, i}-\frac{\partial \psi}{\partial z_{\alpha, j}} f_{\alpha}^{0}\right] \dot{\theta}_{, j}
\end{aligned}
$$




$$
\begin{aligned}
& -\frac{1}{c} \frac{\partial \psi}{\partial \theta_{, i}} \frac{\partial e}{\partial \theta_{, j}} \dot{\theta}_{, j i}-\left(\frac{1}{c} \frac{\partial \psi}{\partial \theta_{, i}} \frac{\partial q_{j}}{\partial \theta_{, k}}+\frac{\partial \psi}{\partial w_{A, i}} \frac{\partial \Phi_{A j}}{\partial \theta_{, k}}\right) \theta_{, k j i} \\
& -\frac{1}{c}\left[\left(\frac{\partial \psi}{\partial \theta}+s\right) \frac{\partial e}{\partial z_{\beta}}+\frac{\partial \psi}{\partial \theta_{, i}}\left(\frac{\partial e}{\partial z_{\beta}}\right)_{, i}\right]_{z_{\beta}} \\
& -\frac{1}{c}\left[\left(\frac{\partial \psi}{\partial \theta}+s\right) \frac{\partial e}{\partial z_{\beta, i}}+\frac{\partial \psi}{\partial \theta_{, i}} \frac{\partial e}{\partial z_{\beta}}+\frac{\partial \psi}{\partial \theta_{, k}}\left(\frac{\partial e}{\partial z_{\beta, i}}\right)_{, k}\right] \dot{z}_{\beta, i}-\frac{1}{c} \frac{\partial \psi}{\partial \theta_{, i}} \frac{\partial e}{\partial z_{\beta, k}} \dot{z}_{\beta, k i} \\
& -\left[\frac{1}{c} \frac{\partial \psi}{\partial \theta_{, i}} \frac{\partial q_{j}}{\partial z_{\beta, k}}-\frac{\partial \psi}{\partial z_{\alpha, i}} f_{\alpha \beta k j}^{1}-\frac{\partial \psi}{\partial w_{A, i}} \frac{\partial \Phi_{A j}}{\partial z_{\beta, k}}\right] z_{\beta, k j i} \\
& -\frac{1}{c}\left[\left(\frac{\partial \psi}{\partial \theta}+s\right) \frac{\partial e}{\partial w_{A}}+\frac{\partial \psi}{\partial \theta_{, i}}\left(\frac{\partial e}{\partial w_{A}}\right)_{, i}\right] \dot{w}_{A} \\
& -\frac{1}{c}\left[\frac{\partial \psi}{\partial \theta_{, i}} \frac{\partial e}{\partial w_{A}}+\left(\frac{\partial \psi}{\partial \theta}+s\right) \frac{\partial e}{\partial w_{A, i}}+\frac{\partial \psi}{\partial \theta_{, k}}\left(\frac{\partial e}{\partial w_{A, i}}\right)_{, k}\right] \dot{w}_{A, i}-\frac{1}{c} \frac{\partial \psi}{\partial \theta_{, i}} \frac{\partial e}{\partial w_{A, k}} \dot{w}_{A, k i} \\
& -\left[\frac{1}{c} \frac{\partial \psi}{\partial \theta_{, i}} \frac{\partial q_{j}}{\partial w_{A, k}}-\frac{\partial \psi}{\partial z_{\alpha, i}} f_{\alpha A k j}^{2}+\frac{\partial \psi}{\partial w_{B, i}} \frac{\partial \Phi_{B j}}{\partial w_{A, k}}\right] w_{A, k j i} \\
& +\Im\left(\theta, \theta_{, i}, \theta_{, j i}, z_{\alpha}, z_{\alpha, i}, z_{\alpha, j i}, w_{A}, w_{A, i}, w_{A, j i}\right) \geq 0,
\end{aligned}
$$

with $\mathfrak{s}$ a suitable regular function of its arguments.

The above inequality is linear in the time derivatives which cannot be expressed as functions of the elements of the state space, and in the gradients which are two orders higher than the gradients in the state space. However, it is not linear in the gradients which are one order higher than the gradients in the state space. Hence, we have to distinguish between higher derivatives and highest derivatives (Cimmelli et al. 2009b). The higher derivatives are the spatial derivatives whose order is higher than that one of the gradients entering the state space. The highest derivatives are both the time derivatives of the elements of the state space which cannot be expressed as functions of the basic fields and the higher derivatives whose order is the highest one.

In order to derive the consequences of equation (2.6), first we note that the highest derivatives entering equation (2.6) are the following: $\dot{\theta}, \dot{\theta}_{, j}, \dot{\theta}_{, j i}, \theta_{, k j i}, \dot{z}_{\beta}, \dot{z}_{\beta, i}$, $\dot{z}_{\beta, k i}, z_{\beta, k j i}, \dot{w}_{A}, \dot{w}_{A, i}, \dot{w}_{A, k i}, w_{A, k j i}$. In any point $\left(P_{0}, t_{0}\right) \in \mathbb{R}^{3} \times[0,+\infty[$, the value of these derivatives is completely arbitrary and independent of their coefficients into the brackets. Then, should even only one of these brackets be different from zero, the entropy inequality could be easily violated. As a consequence, all the brackets must vanish and this yields the thermodynamic restrictions

$$
\begin{gathered}
\frac{\partial \psi}{\partial z_{\alpha}} f_{\alpha}^{0}+\frac{\partial \psi}{\partial z_{\alpha, i}}\left(f_{\alpha}^{0}\right)_{, i}-\frac{1}{c} \frac{\partial \psi}{\partial \theta_{, i}}\left(\frac{\partial e}{\partial \theta}\right)_{, i}=0 \\
-\frac{1}{c}\left(\frac{\partial \psi}{\partial \theta}+s\right) \frac{\partial e}{\partial \theta_{, j}}-\frac{1}{c} \frac{\partial \psi}{\partial \theta_{, i}}\left(\frac{\partial e}{\partial \theta_{, j}}\right)_{, i}+\frac{\partial \psi}{\partial z_{\alpha, j}} f_{\alpha}^{0}=0
\end{gathered}
$$




$$
\begin{gathered}
\frac{\partial \psi}{\partial \theta_{, i}} \frac{\partial e}{\partial \theta_{, j}}=0 \\
\frac{1}{c} \frac{\partial \psi}{\partial \theta_{, i}} \frac{\partial q_{j}}{\partial \theta_{, k}}+\frac{\partial \psi}{\partial w_{A, i}} \frac{\partial \Phi_{A j}}{\partial \theta_{, k}}=0 \\
\left(\frac{\partial \psi}{\partial \theta}+s\right) \frac{\partial e}{\partial z_{\beta}}+\frac{\partial \psi}{\partial \theta_{, i}}\left(\frac{\partial e}{\partial z_{\beta}}\right)_{, i}=0 \\
\left(\frac{\partial \psi}{\partial \theta}+s\right) \frac{\partial e}{\partial z_{\beta, i}}+\frac{\partial \psi}{\partial \theta_{, i}} \frac{\partial e}{\partial z_{\beta}}+\frac{\partial \psi}{\partial \theta_{, k}}\left(\frac{\partial e}{\partial z_{\beta, i}}\right)_{, k}=0 \\
\frac{\partial \psi}{\partial \theta_{, i}} \frac{\partial e}{\partial z_{\beta, k}}=0, \\
-\frac{1}{c} \frac{\partial \psi}{\partial \theta_{, i}} \frac{\partial q_{j}}{\partial z_{\beta, k}}+\frac{\partial \psi}{\partial z_{\alpha, i}} f_{\alpha \beta k j}^{1}+\frac{\partial \psi}{\partial w_{A, i}} \frac{\partial \Phi_{A j}}{\partial z_{\beta, k}}=0 \\
\left(\frac{\partial \psi}{\partial \theta}+s\right) \frac{\partial e}{\partial w_{A}}+\frac{\partial \psi}{\partial \theta_{, i}}\left(\frac{\partial e}{\partial w_{A}}\right)_{, i}=0, \\
\frac{\partial \psi}{\partial \theta_{, i}} \frac{\partial e}{\partial w_{A}}+\left(\frac{\partial \psi}{\partial \theta}+s\right) \frac{\partial e}{\partial w_{A, i}}+\frac{\partial \psi}{\partial \theta_{, k}}\left(\frac{\partial e}{\partial w_{A, i}}\right)_{, k}=0, \\
\frac{\partial \psi}{\partial \theta_{, i}} \frac{\partial e}{\partial w_{A, k}}=0, \\
\frac{1}{c} \frac{\partial \psi}{\partial \theta_{, i}} \frac{\partial q_{j}}{\partial w_{A, k}}+\frac{\partial \psi}{\partial z_{\alpha, i}} f_{\alpha A k j}^{2}-\frac{\partial \psi}{\partial w_{B, i}} \frac{\partial \Phi_{B j}}{\partial w_{A, k}}=0
\end{gathered}
$$

and

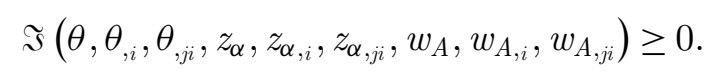

Remark. In our analysis, we did not require that the system of equations (1.2), (2.2) and (2.3) admits analytic solutions, but simply that the Cauchy problem for it is well posed in a suitable functional space (Ruggeri 1988). Hence, we avoided any additional hypothesis which requires a strong regularity of the solution as, for instance, the symmetry of higher-order spatial derivatives of the unknown fields. In the case of analytic solutions, a set of weaker restrictions, involving only the symmetric part of the coefficients of the highest derivatives $\dot{\theta}_{, j i}, \theta_{, k j i}, \dot{z}_{\beta, k i}, z_{\beta, k j i}$, $\dot{w}_{A, k i}, w_{A, k j i}$, would be true.

A further important difference with respect to the classical case is that in equation (2.6), the coefficients of the highest derivatives include terms that, in principle, may also contain some higher-order derivatives of the state functions. These coefficients must vanish anyway, as they are independent of the highest derivatives. However, we must require that the consequent restrictions involve only the constitutive equations. A direct inspection of equations (2.7)-(2.18) shows that this is true if the functions $f_{\alpha}^{0}$ are local and, moreover, if either

$$
\psi=\psi\left(\theta, z_{\alpha}, z_{\alpha, i}, w_{A}, w_{A, i}\right)
$$

or

$$
e=e\left(\theta, z_{\alpha}, w_{A}\right) .
$$


Let us suppose equation (2.20) holds. In such a case, the set of thermodynamic restriction equations $(2.7)-(2.18)$ yields

$$
\begin{gathered}
\frac{\partial \psi}{\partial z_{\alpha}} f_{\alpha}^{0}+\frac{\partial \psi}{\partial z_{\alpha, i}}\left(f_{\alpha}^{0}\right)_{, i}=0 \\
-\frac{1}{c}\left(\frac{\partial \psi}{\partial \theta}+s\right) \frac{\partial e}{\partial \theta_{, j}}+\frac{\partial \psi}{\partial z_{\alpha, j}} f_{\alpha}^{0}=0 \\
\frac{\partial \psi}{\partial w_{A, i}} \frac{\partial \Phi_{A j}}{\partial \theta_{, k}}=0 \\
\left(\frac{\partial \psi}{\partial \theta}+s\right) \frac{\partial e}{\partial z_{\beta}}=0 \\
\left(\frac{\partial \psi}{\partial \theta}+s\right) \frac{\partial e}{\partial z_{\beta, i}}=0 \\
\frac{\partial \psi}{\partial z_{\alpha, i}} f_{\alpha \beta k j}^{1}+\frac{\partial \psi}{\partial w_{A, i}} \frac{\partial \Phi_{A j}}{\partial z_{\beta, k}}=0 \\
\left(\frac{\partial \psi}{\partial \theta}+s\right) \frac{\partial e}{\partial w_{A}}=0 \\
\left(\frac{\partial \psi}{\partial \theta}+s\right) \frac{\partial e}{\partial w_{A, i}}=0 \\
\frac{\partial \psi}{\partial z_{\alpha, i}} f_{\alpha A k j}^{2}-\frac{\partial \psi}{\partial w_{B, i}} \frac{\partial \Phi_{B j}}{\partial w_{A, k}}=0
\end{gathered}
$$

and

From the restrictions above, if the internal energy depends at least on one of the variables $z_{\beta}, z_{\beta, i}, w_{A}, w_{A, i}$, then the classical relation

$$
s=-\frac{\partial \psi}{\partial \theta}
$$

ensues.

On the other hand, if the condition (2.21) holds, we obtain

$$
\begin{gathered}
\frac{\partial \psi}{\partial z_{\alpha}} f_{\alpha}^{0}+\frac{\partial \psi}{\partial z_{\alpha, i}}\left(f_{\alpha}^{0}\right)_{, i}-\frac{1}{c} \frac{\partial \psi}{\partial \theta_{, i}}\left(\frac{\partial e}{\partial \theta}\right)_{, i}=0, \\
\frac{\partial \psi}{\partial z_{\alpha, j}} f_{\alpha}^{0}=0 \\
\frac{1}{c} \frac{\partial \psi}{\partial \theta_{, i}} \frac{\partial q_{j}}{\partial \theta_{, k}}+\frac{\partial \psi}{\partial w_{A, i}} \frac{\partial \Phi_{A j}}{\partial \theta_{, k}}=0 \\
\left(\frac{\partial \psi}{\partial \theta}+s\right) \frac{\partial e}{\partial z_{\beta}}=0 \\
\frac{\partial \psi}{\partial \theta_{, i}} \frac{\partial e}{\partial z_{\beta}}=0
\end{gathered}
$$


and

$$
\begin{gathered}
-\frac{1}{c} \frac{\partial \psi}{\partial \theta_{, i}} \frac{\partial q_{j}}{\partial z_{\beta, k}}+\frac{\partial \psi}{\partial z_{\alpha, i}} f_{\alpha \beta k j}^{1}+\frac{\partial \psi}{\partial w_{A, i}} \frac{\partial \Phi_{A j}}{\partial z_{\beta, k}}=0, \\
\left(\frac{\partial \psi}{\partial \theta}+s\right) \frac{\partial e}{\partial w_{A}}+\frac{\partial \psi}{\partial \theta_{, i}}\left(\frac{\partial e}{\partial w_{A}}\right)_{, i}=0 \\
\frac{\partial \psi}{\partial \theta_{, i}} \frac{\partial e}{\partial w_{A}}=0 \\
-\frac{1}{c} \frac{\partial \psi}{\partial \theta_{, i}} \frac{\partial q_{j}}{\partial w_{A_{, k}}}+\frac{\partial \psi}{\partial z_{\alpha, i}} f_{\alpha A k j}^{2}-\frac{\partial \psi}{\partial w_{B, i}} \frac{\partial \Phi_{B j}}{\partial w_{A, k}}=0 .
\end{gathered}
$$

Hence, the conditions (2.30) and (2.31) hold again unless $e$ depends on the absolute temperature only. If this is not the case, by the restrictions above we infer

and

$$
\begin{gathered}
\frac{\partial \psi}{\partial z_{\alpha}} f_{\alpha}^{0}+\frac{\partial \psi}{\partial z_{\alpha, i}}\left(f_{\alpha}^{0}\right)_{, i}=0, \\
\frac{\partial \psi}{\partial z_{\alpha, j}} f_{\alpha}^{0}=0 \\
\frac{\partial \psi}{\partial w_{A, i}} \frac{\partial \Phi_{A j}}{\partial \theta_{, k}}=0 \\
\frac{\partial \psi}{\partial z_{\alpha, i}} f_{\alpha \beta k j}^{1}+\frac{\partial \psi}{\partial w_{A, i}} \frac{\partial \Phi_{A j}}{\partial z_{\beta, k}}=0 \\
\frac{\partial \psi}{\partial z_{\alpha, i}} f_{\alpha A k j}^{2}-\frac{\partial \psi}{\partial w_{B, i}} \frac{\partial \Phi_{B j}}{\partial w_{A, k}}=0 .
\end{gathered}
$$

Let us suppose for a while that the intensive variable $\mathbf{z}$ does not enter the state space. From equations (2.41)-(2.45), it follows

$$
\frac{\partial \psi}{\partial w_{A, i}} \frac{\partial \Phi_{A j}}{\partial \theta_{, k}}=0
$$

and

$$
\frac{\partial \psi}{\partial w_{B, i}} \frac{\partial \Phi_{B j}}{\partial w_{A, k}}=0 .
$$

The previous restrictions can be trivially satisfied if either $\psi$ or $\Phi_{A}$ is local. A similar result has been obtained by Cimmelli (2007) and referred to as law of the exchange of non-locality.

The residual inequality (2.19) still depends on the higher derivatives $\theta_{, j i}, z_{\alpha, j i}$ and $w_{A, j i}$ and its form is peculiar of the extended procedure. Indeed, if we denote by $X_{\Delta}, \Delta=1, \ldots, \bar{S}=9(1+\bar{N}+\bar{M})$, the generic element of the set of independent higher derivatives, the inequality above can be written as (Cimmelli et al. 2009b)

$$
A_{\Delta \Omega} X_{\Delta} X_{\Omega}+B_{\Delta} X_{\Delta}+C \geq 0
$$


where $A_{\Delta \Omega}, B_{\Delta}$ and $C$ depend on the state functions only. Second law requires the inequality (2.48) to be satisfied for arbitrary values of $X_{\Delta}$. It is worth observing that the first term in relation (2.48) can assume a definite sign for arbitrary values of the $X_{\Delta}$. To this end, it suffices that the matrix $A_{\Delta \Omega}$ is positive semidefinite. The same is not true for the linear terms in $X_{\Delta}$, as the coefficients $B_{\Delta}$ are independent of the $X_{\Delta}$. Hence, for $B_{\Delta} \neq 0$ and arbitrary $X_{\Delta}$, the quantity $B_{\Delta} X_{\Delta}$ could take arbitrary negative values, and the inequality (2.48) could be violated. Then, the additional restrictions

$$
B_{\Delta}=0, \quad A_{\Delta \Omega} X_{\Delta} X_{\Omega}+C \geq 0
$$

ensue. Putting $B_{\Delta}=0$, we get the additional set of thermodynamic restrictions

$$
\begin{gathered}
-\frac{\partial \psi}{\partial w_{A}} \frac{\partial \Phi_{A i}}{\partial \theta_{, k}}-\frac{\partial \psi}{\partial w_{A, i}}\left(\frac{\partial \Phi_{A k}}{\partial \theta}+\frac{\partial^{2} \Phi_{A j}}{\partial \theta \partial \theta_{, k}} \theta_{, j}+\frac{\partial^{2} \Phi_{A j}}{\partial z_{\beta} \partial \theta_{, k}} z_{\beta, j}+\frac{\partial^{2} \Phi_{A j}}{\partial w_{B} \partial \theta_{, k}} w_{B, j}\right) \\
-\frac{\partial \psi}{\partial w_{A, j}}\left(\frac{\partial^{2} \Phi_{A i}}{\partial \theta_{, k} \partial \theta} \theta_{, j}+\frac{\partial^{2} \Phi_{A i}}{\partial \theta_{, k} \partial z_{\beta}} z_{\beta, j}+\frac{\partial^{2} \Phi_{A i}}{\partial \theta_{, k} \partial w_{B}} w_{B, j}\right)=0 \\
\frac{\partial \psi}{\partial z_{\alpha}} f_{\alpha \beta k i}^{1}+\frac{\partial \psi}{\partial z_{\alpha, j}}\left(\frac{\partial f_{\alpha \beta k i}^{1}}{\partial \theta} \theta_{, j}+\frac{\partial f_{\alpha \beta k i}^{1}}{\partial z_{\gamma}} z_{\gamma, j}+\frac{\partial f_{\alpha \beta k i}^{1}}{\partial w_{A}} w_{A, j}\right)-\frac{\partial \psi}{\partial w_{A}} \frac{\partial \Phi_{A i}}{\partial z_{\beta, k}} \\
-\frac{\partial \psi}{\partial w_{A, i}}\left(\frac{\partial \Phi_{A k}}{\partial z_{\beta}}+\frac{\partial^{2} \Phi_{A j}}{\partial \theta \partial z_{\beta, k}} \theta_{, j}+\frac{\partial^{2} \Phi_{A j}}{\partial z_{\gamma} \partial z_{\beta, k}} z_{\gamma, j}+\frac{\partial^{2} \Phi_{A j}}{\partial w_{B} \partial z_{\beta, k}} w_{B, j}\right) \\
\quad-\frac{\partial \psi}{\partial w_{A, j}}\left(\frac{\partial^{2} \Phi_{A i}}{\partial z_{\beta, k} \partial \theta} \theta_{, j}+\frac{\partial^{2} \Phi_{A i}}{\partial z_{\beta, k} \partial z_{\gamma}} z_{\gamma, j}+\frac{\partial^{2} \Phi_{A i}}{\partial z_{\beta, k} \partial w_{B}} w_{B, j}\right)=0 \\
\frac{\partial \psi}{\partial z_{\alpha}} f_{\alpha B k i}^{2}+\frac{\partial \psi}{\partial z_{\alpha, j}}\left(\frac{\partial f_{\alpha B k i}^{2}}{\partial \theta} \theta_{, j}+\frac{\partial f_{\alpha B k i}^{2}}{\partial z_{\beta}} z_{\beta, j}+\frac{\partial f_{\alpha B k i}^{2}}{\partial w_{A}} w_{A, j}\right)-\frac{\partial \psi}{\partial w_{A}} \frac{\partial \Phi_{A i}}{\partial w_{B, k}} \\
-\frac{\partial \psi}{\partial w_{A, i}}\left(\frac{\partial \Phi_{A k}}{\partial w_{B}}+\frac{\partial^{2} \Phi_{A j}}{\partial \theta \partial w_{B, k}} \theta_{, j}+\frac{\partial^{2} \Phi_{A j}}{\partial z_{\beta} \partial w_{B, k}} z_{\beta, j}+\frac{\partial^{2} \Phi_{A j}}{\partial w_{C} \partial w_{B, k}} w_{C, j}\right) \\
-\frac{\partial \psi}{\partial w_{A, j}}\left(\frac{\partial^{2} \Phi_{A i}}{\partial w_{B, k} \partial \theta} \theta_{, j}+\frac{\partial^{2} \Phi_{A i}}{\partial w_{B, k} \partial z_{\beta}} z_{\gamma, j}+\frac{\partial^{2} \Phi_{A i}}{\partial w_{B, k} \partial w_{C}} w_{C, j}\right)=0
\end{gathered}
$$

In the simpler case in which $\psi$ is local, equations (2.50)-(2.52) reduce to

and

$$
\begin{gathered}
\frac{\partial \psi}{\partial w_{A}} \frac{\partial \Phi_{A j}}{\partial \theta_{, k}}=0 \\
\frac{\partial \psi}{\partial z_{\alpha}} f_{\alpha \beta k i}^{1}-\frac{\partial \psi}{\partial w_{A}} \frac{\partial \Phi_{A i}}{\partial z_{\beta, k}}=0
\end{gathered}
$$

Finally, if $f_{\alpha \beta k i}^{1}$ and $f_{\alpha \beta k i}^{2}$ vanish, the restrictions above can be trivially satisfied if either $\psi=\psi\left(\theta, z_{\alpha}\right)$, or $\Phi_{A}$ is local. 


\section{Dynamical temperature and nonlinear heat transport equation}

The analysis of diffusive-relaxational heat conduction in solids (Ackerman \& Guyer 1968), or in miniaturized systems (Chen 2001; Jou et al. 2005; Alvarez \& Jou 2007), has given a strong impulse to the recent development of nonequilibrium thermodynamics. Such a phenomenon cannot be described by the classical Fourier law because it is not capable to reproduce the relaxational regime (Cattaneo 1948). In the linear approximation, the most celebrated diffusiverelaxational heat transport equation is the Guyer-Krumhansl one (Guyer \& Krumhansl 1966)

$$
\tau_{\mathrm{R}} \dot{q}_{i}+q_{i}=-\kappa \theta_{, i}+\frac{9}{5} \frac{\kappa \tau_{\mathrm{N}}}{c}\left(q_{i_{, k k}}+2 q_{k, k i}\right),
$$

with $\kappa$ as the heat conductivity. Moreover, $\tau_{\mathrm{N}}$ and $\tau_{\mathrm{R}}$ are two relaxation times related, respectively, to normal and resistive scattering processes of the heat carriers (Reissland 1973). If $\tau_{\mathrm{N}}$ is negligible, it reduces to the Maxwell-Cattaneo equation (Cattaneo 1948)

$$
\tau_{\mathrm{R}} \dot{q}_{i}+q_{i}=-\kappa \theta_{, i},
$$

which describes the second-sound propagation. Nowadays, several macroscopic theories have been developed to cope with the above equations (Joseph \& Preziosi 1989, 1990; Müller \& Ruggeri 1998; Jou et al. 2001). Here, we consider the theory of heat conduction with a non-equilibrium semi-empirical temperature (Cimmelli \& Kosiński 1991; Cimmelli \& Frischmuth 2005; Cimmelli et al. 2009a). In such a theory, an additional internal scalar state variable $\beta$ (the semi-empirical temperature scale) and an evolution equation for it was introduced. The physical interpretation of $\beta$ is of a dynamical non-equilibrium temperature such that the heat flux is in the inverted direction of the gradient of $\beta$ (Cimmelli \& Kosiński 1991; Cimmelli et al. 2009a), namely

$$
q_{i}=-\kappa \beta_{, i} .
$$

In setting up our model, we suppose that the state space $Z$ is spanned by the absolute temperature $\theta$, the semi-empirical temperature $\beta$, together with their first-order gradients $\nabla \theta$ and $\nabla \beta$, namely

$$
Z=\left\{\theta, \theta_{, i}, \beta, \beta_{, i}\right\} .
$$

In an equilibrium system, the use of both temperatures would be redundant. However, out of equilibrium, the absolute temperature $\theta$ is not distributed as in equilibrium and, therefore, $\beta$ is a truly independent quantity, not redundant with $\theta$ (Cimmelli \& Kosiński 1991; Cimmelli et al. 2009a). We suppose that the semi-empirical temperature $\beta$ is governed by the evolution equation

$$
\dot{\beta}=f_{0}(Z)+f_{j i}^{\beta}(Z) \beta_{, j i}+f_{j i}^{\theta}(Z) \theta_{, j i},
$$

where $f_{0}, f_{j i}^{\beta}$ and $f_{j i}^{\theta}$ are suitable tensorial functions. The evolution equation for the absolute temperature $\theta$, in this particular case, reads

$$
c \dot{\theta}=-\left(\frac{\partial e}{\partial \beta} \dot{\beta}+\frac{\partial e}{\partial \theta_{, i}} \dot{\theta}_{, i}+\frac{\partial e}{\partial \beta_{, i}} \dot{\beta}_{, i}+\frac{\partial q_{i}}{\partial \theta} \theta_{, i}+\frac{\partial q_{i}}{\partial \beta} \beta_{, i}+\frac{\partial q_{i}}{\partial \theta_{, k}} \theta_{, k i}+\frac{\partial q_{i}}{\partial \beta_{, k}} \beta_{, k i}\right),
$$


and the inequality (1.1) reduces to

$$
\left(\frac{\partial \psi}{\partial \theta}+s\right) \dot{\theta}+\frac{\partial \psi}{\partial \beta} \dot{\beta}+\frac{\partial \psi}{\partial \theta_{, i}} \dot{\theta}_{, i}+\frac{\partial \psi}{\partial \beta_{, i}} \dot{\beta}_{, i}+\frac{1}{\theta} q_{i} \theta_{, i} \leq 0 .
$$

If we restrict ourselves to thermodynamic processes corresponding to analytic solutions of the basic equations (3.4) and (3.5), and study the consequences of the inequality (3.6) by the procedure illustrated in the previous section, we are led to the set of thermodynamic restrictions

$$
\begin{gathered}
\frac{\partial \psi}{\partial \beta}+\frac{\partial \psi}{\partial \beta_{, j}} f_{0_{j}}-\frac{1}{c} \frac{\partial \psi}{\partial \theta_{, j}}\left(\frac{\partial e}{\partial \theta}\right)_{, j}=0, \\
\left(\frac{\partial \psi}{\partial \theta}+s\right) \frac{\partial e}{\partial \theta_{, i}}+\frac{\partial \psi}{\partial \theta_{, j}}\left(\frac{\partial e}{\partial \theta_{, i}}\right)_{, j}+\frac{\partial \psi}{\partial \beta_{, i}} f_{0}=0, \\
\left\langle\frac{\partial \psi}{\partial \theta_{, j}} \frac{\partial e}{\partial \theta_{, i}}\right\rangle=0, \\
\left\langle\frac{1}{c} \frac{\partial \psi}{\partial \theta_{, j}} \frac{\partial q_{i}}{\partial \theta_{, k}}-\frac{\partial \psi}{\partial \beta_{, j}} f_{k i}^{\theta}\right\rangle=0, \\
\left(\frac{\partial \psi}{\partial \theta}+s\right) \frac{\partial e}{\partial \beta}+\frac{\partial \psi}{\partial \theta_{, j}}\left(\frac{\partial e}{\partial \beta}\right)_{, j}=0, \\
+s) \frac{\partial e}{\partial \beta_{, i}}+\frac{\partial \psi}{\partial \theta_{, j}}\left(\frac{\partial e}{\partial \beta_{, i}}\right)_{, j}+\frac{\partial \psi}{\partial \theta_{, i}} \frac{\partial e}{\partial \beta}=0, \\
\left\langle\frac{\partial \psi}{\partial \theta_{, j}} \frac{\partial e}{\partial \beta_{, i}}\right\rangle=0, \\
\left\langle\frac{\partial \psi}{c} \frac{\partial \psi}{\partial \theta_{, j}} \frac{\partial q_{i}}{\partial \beta_{, k}}-\frac{\partial \psi}{\partial \beta_{, j}} f_{k i}^{\beta}\right\rangle=0 \\
\left\langle\frac{\partial \psi}{\partial \beta_{, j}} f_{k i}^{\theta}\right\rangle=0 .
\end{gathered}
$$

and

In the above equations the symbols $\left\langle a_{i j}\right\rangle$ and $\left\langle a_{i j k}\right\rangle$ denote the symmetrized part of second-order and third-order tensors $a_{i j}$ and $a_{i j k}$ with respect to the indices $(i, j)$ and $(i, j, k)$, respectively.

The restrictions above can be satisfied in several ways. Below, we analyse a possible solution, leading to meaningful consequences on the model. Hence, let us choose

$$
\begin{gathered}
f_{0}=\frac{1}{\tau_{\mathrm{R}}}(\theta-\beta), \\
\frac{\partial \psi}{\partial \theta_{, j}}=0,
\end{gathered}
$$


and

$$
\begin{gathered}
f_{k i}^{\theta}=0 \\
f_{m i}^{\beta}=-\frac{\kappa}{A c} \frac{\beta}{T} \delta_{m i},
\end{gathered}
$$

where $A$ is a constant, and $T$ means the value of the semi-empirical temperature at the equilibrium $(\dot{\beta}=0)$. Then, from the set of thermodynamic restrictions (3.7)-(3.15), it follows

and

$$
\begin{gathered}
\frac{\partial \psi}{\partial \beta}+\frac{\partial \psi}{\partial \beta_{, j}} f_{0, j}=0 \\
\left(\frac{\partial \psi}{\partial \theta}+s\right) \frac{\partial e}{\partial \theta_{, i}}+\frac{\partial \psi}{\partial \beta_{, i}} f_{0}=0, \\
\left(\frac{\partial \psi}{\partial \theta}+s\right) \frac{\partial e}{\partial \beta}=0 \\
\left(\frac{\partial \psi}{\partial \theta}+s\right) \frac{\partial e}{\partial \beta_{, i}}=0 \\
\frac{\kappa}{A c} \frac{\beta}{T}\left\langle\frac{\partial \psi}{\partial \beta_{, j}} \delta_{k i}\right\rangle=0
\end{gathered}
$$

By equation (3.23), we get

$$
s=-\frac{\partial \psi}{\partial \theta},
$$

which makes equation (3.22) satisfied. Then, under the assumption

$$
\frac{1}{A}=\frac{27}{5} \frac{\tau_{\mathrm{N}}}{\tau_{\mathrm{R}}}
$$

the evolution equation (3.4) for $\beta$ becomes

$$
\dot{\beta}=\frac{1}{\tau_{\mathrm{R}}}(\theta-\beta)+\frac{27}{5} \frac{\kappa \tau_{\mathrm{N}}}{c \tau_{\mathrm{R}}} \frac{\beta}{T} \beta_{, j j} .
$$

Finally, once the balance of energy (1.2), the constitutive equation (3.3) for the heat flux and the symmetry of the third-order spatial derivatives of $\beta$ are taken into account, if the thermophysical material functions are constant, the gradient extension of the above equation leads to the nonlinear heat transport equation

$$
\tau_{\mathrm{R}} \dot{q}_{i}+\lambda q_{i}=-\kappa \theta_{, i}+l^{2}\left(q_{i_{k k}}+2 q_{k_{, k i}}\right),
$$

where $l^{2}=(9 / 5)\left(\kappa \tau_{\mathrm{N}} / c\right)(\beta / T)$, and $\lambda=1+(27 / 5)\left(\tau_{\mathrm{N}} / c T \kappa\right) q_{k, k}$. Such an equation extends to the nonlinear regime the Guyer-Krumhansl equation (3.1), which can be recovered by equation (3.27) in homogeneous states close to the thermal equilibrium, namely when $\dot{e}, \dot{\beta}, \nabla e$ and $\nabla \beta$ are negligible. It is worth observing that in equation (3.27) two different types of nonlinearity appear. The first one is due to the dependence of the coefficient $\lambda$ on $\nabla \cdot \mathbf{q}$, and this introduces a second-order term in the heat flux and its spatial derivatives. The second type of nonlinearity, which is often referred to as semi-linearity, is due to the dependence of $l^{2}$ on the dynamical temperature $\beta$, and does not contain second-order terms in the heat flux and its spatial derivatives. 
In the present case, the evolution equation for $\beta$ does not contain the firstorder gradient of $\beta$. This is not the most general case. For instance, a more general governing equation, including a quadratic term in the first gradient of $\beta$, has been considered by Cimmelli et al. (submitted). The thermodynamic analysis of such a case can be developed in the realm of thermodynamics with Lagrange multipliers. In the linear case, it yields the classical Guyer-Krumhansl equation. In the nonlinear case, the heat transport equation is more general than that derived here and contains several quadratic terms in the heat flux and its first and second gradients, whose physical interpretation is not yet clear. To our knowledge, the above derivation is the first one developed by applying the Coleman-Noll procedure in the absence of extra-fluxes.

By straightforward calculations, it is possible to show that if the classical Coleman-Noll procedure would have been applied without modifying either first or second law, our model would have been incompatible with thermodynamics. On the contrary, by applying the extended procedure, we have proven that the model is compatible with the second law, without any 'ad hoc' hypothesis on the basic thermodynamic laws. Moreover, it was capable to dismantle a physical property which does not emerge in the classical case, namely that the entropy and the free energy can depend on the gradients, too.

\section{Conclusions}

In the present paper, we have proposed a generalization of the classical ColemanNoll procedure for the exploitation of the second law of thermodynamics. The new method consists in substituting into the entropy inequality not only the governing equations for the wanted variables, but their gradient extensions too. We have generalized some classical results of rational thermodynamics, by proving that even in the absence of energy or entropy extra-flux, nonlocal materials of arbitrary order are still compatible with the second law. Of course, our result does not imply that these extra-fluxes do not exist but that they are no longer essential to ensure the thermodynamic compatibility. Hence, their effective existence should be decided only on the basis of suitable experimental results.

Indeed, the Coleman-Noll approach is originally based on the fact that entropy source at a boundary is proportional to the heat source at a boundary, and both can be expressed linearly in terms of the unit normal. The first assumption follows from a general idea originating from the works of Clausius, Gibbs and Duhem. The second one, owing to Stokes but following in the path opened by Fourier and Cauchy, is what introduces the vectorial fluxes. On the other hand, this is necessarily related to the notion of first-gradient theory, because only the description of the surface at the first order, its unit normal, is involved. Therefore, this framework, if not generalized, does not cope with constitutive equations including higher-order gradients. Classically, the problem can be circumvented, for instance, by abandoning the hypothesis of proportionality between heat source and entropy source at the boundary, namely by postulating the existence of an entropy extra-flux. However, such an idea does not seem to be applicable to the rigid heat conductors considered in the present paper. In fact, by supposing that the extra-flux is due to long-range 
interactions, one should answer the question why the same is not postulated for the classical case, with the state space spanned by $\theta$ and $\nabla \theta$ only, which is non-local too.

Here, we proposed a different solution, which does not require to modify the physical setup but only the mathematics necessary to analyse it, namely the technique of exploitation of the entropy principle. It is worth observing that, although the present paper is in the realm of rational thermodynamics, our generalized procedure can be applied whenever one is faced with a weakly non-local theory, such as, for instance, extended irreversible thermodynamics (Jou et al. 2001) or classical irreversible thermodynamics (de Groot \& Mazur 1962).

The extended procedure allowed us to build up a macroscopic model leading to a generalization of the celebrated Guyer-Krumhansl equation. This seems to be important not only in the description of the diffusive-relaxational heat transport, but also in the discussion concerning the fundamentals of different thermodynamic theories (Cimmelli in press). In fact, all modern non-equilibrium thermodynamic theories aim to fit the results from kinetic theory (Müller \& Ruggeri 1998; Jou et al. 2001). On the other hand, as the Guyer-Krumhansl equation has been obtained by solving a linearized phonon Boltzmann equation, i.e. in the framework of the kinetic theory, it seems to be important for any thermodynamic theory to provide a model for the Guyer-Krumhansl equation. The result above proves that rational thermodynamics can provide such a model without any amendment to the classical form of the first and second laws. Hence, it leads to important theoretical consequences concerning the basic principles.

In setting up our model, we needed several additional hypotheses on the constitutive quantities. This is due to the fact that, once the thermodynamic compatibility of the constitutive functions has been proven by the extended exploitation procedure, these functions have still to be set:

- according to the hypotheses of phonon gas hydrodynamics (Reissland 1973);

- according to the linear approximation in the solution of phonon Boltzmann equation (Guyer \& Krumhansl 1966).

However, as the hypotheses of $\S 3$ are correct from the mathematical point of view and compatible with the second law of thermodynamics, the model is physically realizable.

\section{References}

Ackerman, C. C. \& Guyer, R. A. 1968 Temperature pulses in dielectric solids. Ann. Phys. 50, 128-185. (doi:10.1016/0003-4916(68)90320-5)

Alvarez, F. X. \& Jou, D. 2007 Memory and nonlocal effects in heat transport. Appl. Phys. Lett. 90, 083109. (doi:10.1063/1.2645110)

Bazant, Z. P. \& Pijauder-Cabot, G. 1987 Nonlocal damage theory. J. Eng. Mech. ASCE 113, 1512-1533. (doi:10.1061/(ASCE)0733-9399(1987)113:10(1512))

Capriz, G. 1989 Continua with microstructure. Springer Tracts in Natural Philosophy, vol. 35. New York, NY: Springer-Verlag.

Cattaneo, C. 1948 Sulla conduzione del calore. Atti Sem. Mat. Fis. Univ. Modena 3, 83-101.

Chen, G. 2001 Ballistic-diffusive heat-conduction equations. Phys. Rev. Lett. 86, 2297-2300. (doi:10.1103/PhysRevLett.86.2297)

Proc. R. Soc. A (2010) 
Cimmelli, V. A. 2007 An extension of Liu procedure in weakly nonlocal thermodynamics. J. Math. Phys. 48, 113510. (doi:10.1063/1.2804753)

Cimmelli, V. A. In press. Different thermodynamic theories and different heat conduction laws. J. Non-Equilib. Thermodyn. 34.

Cimmelli, V. A. \& Frischmuth, K. 2005 Nonlinear effects in thermal wave propagation near zero absolute temperature. Physica B 355, 147-157. (doi:10.1016/j.physb.2004.10.034)

Cimmelli, V. A. \& Frischmuth, K. 2007 Gradient generalization to the extended thermodynamic approach and diffusive-hyperbolic heat conduction. Physica B 400, 257-265. (doi:10.1016/ j.physb.2007.07.019)

Cimmelli, V. A. \& Kosiński, W. 1991 Nonequilibrium semi-empirical temperature in materials with thermal relaxation. Arch. Mech. 43, 753-767.

Cimmelli, V. A., Sellitto, A. \& Jou, D. $2009 a$ Nonlocal effects and second sound in a nonequilibrium steady state. Phys. Rev. B 79, 014303. (doi:10.1103/PhysRevB.79.014303)

Cimmelli, V. A., Sellitto, A. \& Triani V. $2009 b$ A new thermodynamic framework for second-grade Korteweg-type viscous fluids. J. Math. Phys. 50, 053101. (doi:10.1063/1.3129490)

Cimmelli, V. A., Sellitto, A. \& Jou, D. Submitted. Nonequilibrium temperature, heat waves, and nonlinear heat transport equations.

Coleman, B. D. \& Noll, W. 1963 The thermodynamics of elastic materials with heat conduction and viscosity. Arch. Ration. Mech. Anal. 13, 167-178. (doi:10.1007/BF01262690)

de Groot, S. R. \& Mazur, P. 1962 Non-equilibrium thermodynamics. Amsterdam, The Netherlands: North-Holland Publishing Company.

Dunn, J. E. \& Serrin, J. 1985 On the thermomechanics of the interstitial working. Arch. Ration. Mech. Anal. 88, 95-133. (doi:10.1007/BF00250907)

Gurtin, M. E. \& Vargas, A. S. 1971 On the classical theory of reacting fluid mixtures. Arch. Ration. Mech. Anal. 43, 179-197. (doi:10.1007/BF00251451)

Guyer, R. A. \& Krumhansl, J. A. 1966 Solution of the linearized phonon Boltzmann equation. Phys. Rev. 148, 766-778. (doi:10.1103/PhysRev.148.766)

Joseph, D. D. \& Preziosi, L. 1989 Heat waves. Rev. Mod. Phys. 61, 41-73. (doi:10.1103/ RevModPhys.61.41)

Joseph, D. D. \& Preziosi, L. 1990 Addendum to the paper 'Heat waves' [Rev. Mod. Phys., 1989, 61, 41-73]. Rev. Mod. Phys. 62, 375-391. (doi:10.1103/RevModPhys.62.375)

Jou, D., Casas-Vázqez, J. \& Lebon, G. 2001 Extended irreversible thermodynamics, 3rd edn. Berlin, Germany: Springer-Verlag.

Jou, D., Casas-Vázqez, J., Lebon, G. \& Grmela, M. 2005 A phenomenological scaling approach for heat transport in nano-system. Appl. Math. Lett. 18, 963-967. (doi:10.1016/j.aml.2004.06.026)

Korteweg, D. J. 1901 Sur la forme qui prennent les equations du mouvement des fluids si l'on tient compte des forces capillaires par des variations de densité. Arch. Néer. Sci. Exactes, Ser. II 6, $1-24$.

Lebon, G., Jou, D., Casas-Vázquez, J. \& Muschik, W. 1998 Weakly nonlocal and nonlinear heat transport in rigid solids. J. Non-Equilib. Thermodyn. 23, 176-191.

Liu, I.-S. 1972 Method of Lagrange multipliers for exploitation of the entropy principle. Arch. Ration. Mech. Anal. 46, 131-138. (doi:10.1007/BF00250688)

Müller, I. 1967 On the entropy inequality. Arch. Ration. Mech. Anal. 26, 118-141. (doi:10.1007/ BF00285677)

Müller, I. \& Ruggeri, T. 1998 Rational extended thermodynamics. Berlin, Germany: Springer-Verlag.

Papenfuss, C. \& Forest S. 2006 Thermodynamical framework for higher grade material theories with internal variables or additional degrees of freedom. J. Non-Equilib. Thermodyn. 31, 319-353. (doi:10.1515/JNETDY.2006.014)

Reissland, J. A. 1973 The physics of phonons. London, UK: John Wiley and Sons.

Ruggeri, T. 1988 Thermodynamics and symmetric hyperbolic systems. In Fascicolo speciale: hyperbolic problems, pp. 167-183. Rend. Sem. Mat. Univ. Pol. Torino.

Truesdell, C. 1984 Rational thermodynamics, 2nd edn. Berlin, Germany: Springer Verlag.

Ván, P. \& Vásárhelyi, B. 2001 Second law of thermodynamics and the failure of rock materials. In Rock mechanics in the national interest, vol. 1 (eds J. P. Tinucci, D. Elsworth \& K. A. Heasley), pp. 767-773. Lisse, The Netherlands: Balkema Publishers. 